\title{
Role for Hedgehog signaling in hepatic stellate cell activation and viability
}

\author{
Jason K Sicklick ${ }^{1,2}$, Yin-Xiong Li $^{1,3,4}$, Steve S Choi ${ }^{1}$, Yi Qi ${ }^{1}$, Wei Chen ${ }^{1}$, Marcia Bustamante ${ }^{5}$, \\ Jiawen Huang ${ }^{1}$, Marzena Zdanowicz ${ }^{1}$, Terese Camp ${ }^{1}$, Michael S Torbenson ${ }^{6}$, \\ Marcos Rojkind ${ }^{5}$ and Anna Mae Diehl ${ }^{1}$ \\ ${ }^{1}$ Division of Gastroenterology and Department of Medicine, Duke University Medical Center, Durham, \\ NC, USA; ${ }^{2}$ Department of Surgery, Johns Hopkins University School of Medicine, Baltimore, MD, USA; \\ ${ }^{3}$ Department of Cell Biology, Duke University Medical Center, Durham, NC, USA; ${ }^{4}$ Department of Pediatrics, \\ Duke University Medical Center, Durham, NC, USA; ${ }^{5}$ Departments of Biochemistry, Molecular Biology, and \\ Pathology, George Washington University Medical Center, Washington, DC, USA and ${ }^{6}$ Department of \\ Pathology, Johns Hopkins University School of Medicine, Baltimore, MD, USA
}

\begin{abstract}
Hepatic stellate cells (HSC) have a complex phenotype that includes both neural and myofibroblastic features. The Hedgehog $(\mathrm{Hh})$ pathway has been shown to direct the fate of neural and myofibroblastic cells during embryogenesis and during tissue remodeling in adults. Therefore, we hypothesized that Hh signaling may regulate the fate of HSC in adults. In this study, we find that freshly isolated stellate cells from adult PatchedlacZ transgenic mice exhibit $\beta$-galactosidase activity, indicating Hh pathway activity. Transcripts of Hh ligands, the Hh pathway receptor, and Hh-regulated transcription factors are expressed by stellate cells from mice, rats, and humans. Transfection experiments in a cell line using a $\mathrm{Hh}$-inducible luciferase reporter demonstrate constitutive Hh pathway activity. Moreover, neutralizing antibodies to Hh increase apoptosis, while viability is restored by treatment with $\mathrm{Hh}$ ligand. In vitro treatment of primary stellate cells with cyclopamine (Cyc), a pharmacologic inhibitor of the Hh pathway, inhibits activation and slightly decreases cell survival, while a single injection of Cyc into healthy adult mice reduces activation of HSC by more than $\mathbf{5 0 \%}$ without producing obvious liver damage. Our findings reveal a novel mechanism, namely the Hh pathway, that regulates the activation and viability of HSC.

Laboratory Investigation (2005) 85, 1368-1380. doi:10.1038/labinvest.3700349; published online 19 September 2005
\end{abstract}

Keywords: cirrhosis; cyclopamine; liver; myofibroblast; neuroendocrine cells; patched

Hepatic stellate cells (HSC) are the major profibrogenic cells in the liver. During liver injury, HSC are activated to a myofibroblastic phenotype. Together with portal fibroblasts and septal myofibroblasts of bone marrow origin, activated HSC produce most of the collagen matrix in injured livers. ${ }^{1}$ Thus, emerging antifibrotic therapies aim to inhibit the accumulation of these fibrogenic cells. A better understanding of the mechanisms that regulate the viability and phenotype of HSC will help to advance this goal because the reversibility of hepatic fibrosis appears to hinge upon the elimination of activated HSC. $^{2}$

HSC are thought to be fibroblastic mesenchymal cells $^{3,4}$ based upon their robust induction of $\alpha$ -

Correspondence: Dr AM Diehl, MD, Division of Gastroenterology, Duke University Medical Center, Snyderman-GSRB I Suite 1073, 595 LaSalle Street, Box 3256, Durham, NC 27710, USA.

E-mail: diehlo04@mc.duke.edu

Received 22 July 2005; accepted 8 August 2005; published online 19 September 2005 smooth muscle actin ( $\alpha$-SMA), matrix molecules, and matrix metalloproteinases during activation. ${ }^{5}$ However, these cells also exhibit many neuroendocrine features, including the expression of synaptophysin, glial fibrillary acidic protein (GFAP), neural cell adhesion molecule, nestin, ${ }^{6}$ neurotrophins, ${ }^{7}$ dopamine- $\beta$-hydroxylase (DBH), and tyrosine hydroxylase. ${ }^{8}$ Like neuroendocrine cells, HSC synthesize a variety of catecholamines and express adrenoreceptors, ${ }^{9}$ as well as receptors for other neurohumoral factors, such as acetylcholine, ${ }^{10}$ neuropeptide $\mathrm{Y}^{11}$ angiotensin, ${ }^{12}$ somatostatin, ${ }^{13}$ and leptin. ${ }^{14}$ These features have prompted speculation that HSC might be derived from neural precursors during development. ${ }^{3}$

Recent research demonstrates overlap between the signals that regulate the fates of neural and myofibroblastic cells within the gastrointestinal tract. In vertebrate embryos, enteric neural crest cells migrate and colonize the gut, where they proliferate and differentiate into neurons and glia of the enteric nervous system. This process is regulated by Sonic 
hedgehog (Shh) acting through its cellular receptor, Patched (Ptc). ${ }^{15}$ This interaction releases Smoothened (Smo) from the inhibitory actions of Ptc and initiates a cascade of intracellular events that culminate in the activation of Gli transcription factors which modulate the expression of target genes, including Ptc and Gli1. ${ }^{16}$ This recent evidence for Hedgehog (Hh) signaling in the gastrointestinal tract complements and extends earlier evidence that $\mathrm{Hh}$ provides neural survival signals that are required in developing and adult brains. ${ }^{17}$ Interestingly, recent studies of transgenic mice treated with a Hh inhibitor prove that this pathway is also critical for morphogenesis of the intestinal crypt-villus axis. ${ }^{18}$ Also, during embryogenesis, subepithelial myofibroblasts and desmin-positive smooth muscle progenitors are the Hh-responsive targets that regulate the modeling of the primitive endoderm in order to generate the morphology of mature intestinal mucosa. ${ }^{19}$ Thus, secreted morphogens of the Hh family regulate the fate of intestinal cells that have either neural or myofibroblastic phenotypes. In the adult intestine, cells that express Hh ligands and the Ptc receptor persist at the base of the crypts. Moreover, intestinal injury is accompanied by a dramatic induction of both ligand and receptor expression, suggesting that the Hh pathway plays a role in the repair of damaged intestinal mucosa during adulthood. ${ }^{20}$

As mentioned earlier, HSC participate in the remodeling of injured livers. Based on the knowledge that HSC exhibit features of both neural and myofibroblastic cells, and the recent aforementioned evidence that the Hh pathway regulates neural and myofibroblastic cells that modulate gut mucosal remodeling, we hypothesized that components of the Hh pathway might be present in HSC and that Hh signaling may also regulate the viability and/or activation of these liver cells. To evaluate our hypothesis, we studied Ptc-lacZ transgenic mice in which $\beta$-galactosidase reports $\mathrm{Hh}$ pathway activity, ${ }^{21}$ wild-type mice with and without exposure to the Hh pathway antagonist, cyclopamine (Cyc), ${ }^{22}$ freshly isolated and cultured mouse HSC, cultured primary rat HSC, a spontaneously transformed human HSC line, ${ }^{23}$ and two well-characterized, clonally derived rat HSC lines. ${ }^{24}$ Our results demonstrate that HSC express Hh ligands and multiple components of the Hh pathway, as well as indicate that $\mathrm{Hh}$ pathway activity prevents apoptosis of HSC and modulates HSC activation. These findings identify novel therapeutic targets that might be used to prevent or reverse hepatic fibrosis.

\section{Materials and methods}

\section{Animal Care}

Adult, male C57BL/6 mice were purchased from Jackson Laboratory (Bar Harbor, ME, USA) and adult, male Ptc-lacZ reporter mice were obtained from Dr PA Beachy (Johns Hopkins University, Baltimore, MD, USA). ${ }^{21}$ Animal experiments fulfilled NIH, Johns Hopkins, and Duke University requirements for humane animal care.

\section{Ptc-lacZ Staining and Reporter Assay}

Detection of $\beta$-galactosidase expression was performed using Ptc-lacZ reporter mice. ${ }^{21}$ Staining for gene expression was performed as previously described using the $\beta$-galactosidase Detection Kit (Promega, Madison, WI, USA). ${ }^{25}$

\section{Isolation of Primary Murine Liver Cells}

The nonparenchymal cell (NPC) fraction was isolated by in situ portal vein perfusion with pronasecollagenase to destroy mature hepatocytes and release NPCs from the liver matrix..$^{24,26,27}$ Viable NPCs were collected by density gradient centrifugation through OptiPrep ${ }^{\mathrm{TM}}$ (Accurate Chemical, Norway), which separates HSCs from NPCs. ${ }^{4,28-30}$ Cell fractions were pooled from 2-12 mice. Greater than $95 \%$ of the isolated HSC exhibited autofluorescence typical of quiescent cells, as has been reported by others. $^{30}$ Viable cells were determined by Trypan Blue (GIBCO/BRL, Grand Island, NY, USA) exclusion and counted with a hemocytometer. Freshly isolated HSC were used for RNA analysis or cultured on plastic dishes for up to 7 days in $10 \%$ serum-supplemented RPMI 1640 medium (GIBCO/ BRL) and $10 \mathrm{mM}$ HEPES.

\section{Rat Hepatic Stellate Cell Isolation and Culture}

Primary rat HSC were isolated through Percoll gradient (Amersham Biosciences, Piscataway, NJ, USA), cultured for 10 days, and passaged twice as described previously. ${ }^{24,31,32}$

\section{Culture of Rat and Human Hepatic Stellate Cell Lines}

Clonally derived rat HSC lines ${ }^{24}$ were cultured in $10 \%$ serum-supplemented RPMI 1640 medium (GIBCO/BRL) and $10 \mathrm{mM}$ HEPES. The human LX-2 HSC line was obtained from Dr SL Friedman (Mount Sinai School of Medicine, New York, NY, USA) ${ }^{23}$ and cultured in $2 \%$ serum-supplemented DMEM: Ham's F-12 (1:1, GIBCO/BRL).

\section{Pharmacological Regulation of Hh Signaling}

Cell lines and primary HSC were treated with regulators of $\mathrm{Hh}$ signaling in a dose-dependent fashion. Cultured cell lines were treated with recombinant N-terminus Sonic hedgehog (Shh-N) ligand (10-40 nM, Stem Cell Technologies, Canada). Lines were also treated with mouse IgG $_{1}$ isotype control antibody (R\&D Systems, Minneapolis, MN, 
USA) or 5E1 Hh-neutralizing antibody (University of Iowa Developmental Studies Hybridoma Bank, Iowa City, IA, USA) at concentrations of $0.1-10 \mu \mathrm{g} / \mathrm{ml} .^{33}$ The primary HSC were also treated with Cyc (Calbiochem, San Diego, CA, USA), or its catalytically inactive analog, tomatidine $(3 \mu \mathrm{M}){ }^{22,34}$ All experiments were performed in fetal bovine serum (FBS).

\section{Immunofluorescent Staining of Cultured Cells}

Following 7 days of culture, the media was removed from cultured mouse HSC. The adherent cells were rinsed once in phosphate-buffered saline (PBS). Cells were then fixed with $4 \%$ paraformaldehyde (Sigma) at room temperature for $15 \mathrm{~min}$. The fixed cells were rinsed in $0.1 \mathrm{M}$ Tris-HCl $(\mathrm{pH}$ 7.6, SigmaAldrich):0.05\% Tween-20 (Bio-Rad, Hercules, CA, USA) for $5 \mathrm{~min}, 0.1 \mathrm{M}$ Tris-HCl for $5 \mathrm{~min}, 0.1 \mathrm{M}$ Tris$\mathrm{HCl}: 2 \% \mathrm{FBS}$ for $5 \mathrm{~min}$, and then incubated with monoclonal anti-mouse $\alpha$-SMA antibody (1:5000, Sigma-Aldrich) ${ }^{35}$ for $30 \mathrm{~min}$ at room temperature. Following incubation with primary antibody, the cells were serially rinsed in Tris-HCl, and incubated with the secondary antibody, FITC-conjugated goat anti-mouse $\operatorname{IgG}_{\gamma 2 \mathrm{a}}$ (1:75, Molecular Probes, Carlsbad, CA, USA) for $45 \mathrm{~min}$ at room temperature. After rinsing in $0.1 \mathrm{M}$ Tris-HCl:0.05\% Tween-20 followed by $0.1 \mathrm{M}$ Tris-HCl for $5 \mathrm{~min}$ each, nuclear staining was performed with DAPI diluted in methanol (1:1000, Sigma-Aldrich) for $5 \mathrm{~min}$. The cells were then rinsed with $100 \%$ methanol and photographed. Negative controls were performed by omitting the primary antibody from the protocol.

\section{Immunofluorescent Staining of Mouse Liver}

Serial sections of formalin-fixed, paraffin-embedded mouse livers, 7- $\mu \mathrm{m}$ thick, were used for immunofluorescent staining. Slides were deparaffinized in xylene and serially rehydrated in sequential ethanol (100-70\%). For GFAP immunofluorescence, epitope retrieval was performed by microwave incubating slides in $1 \times$ Antigen Retrieval Citra Solution (BioGenex, San Ramon, CA, USA) for $10 \mathrm{~min}$. For both GFAP and $\alpha$-SMA staining, the slides were treated with $0.1 \mathrm{M}$ Tris-HCl (pH 7.6):0.05\% Tween20 for $5 \mathrm{~min}$, incubated with $1 \mu \mathrm{g} / \mathrm{ml}$ Proteinase $\mathrm{K}$ (Macherey-Nagel, Easton, PA, USA) for $15 \mathrm{~min}$ at room temperature, and then rinsed in water for $10 \mathrm{~min}$. The remainder of staining protocol was the same as for the immunofluorescent staining of cultured cells, except that the primary antibody against GFAP was rabbit anti-bovine GFAP (1:1000, DakoCytomation, Carpinteria, CA, USA), which crossreacts with mouse GFAP. ${ }^{36}$ The secondary antibody was FITC-conjugated goat anti-rabbit IgG (1:50, Molecular Probes, Carlsbad, CA, USA). Slides were coverslipped after the completion of staining. Negative controls were performed by omitting the primary antibody from the protocol.

\section{Cell Viability and Apoptosis Assays}

Cell viability was measured with the Cell Counting Kit-8 (Dojindo Molecular Technologies, Gaithersburg, MD, USA) ${ }^{8,37}$ Briefly, HSC lines were cultured for $24 \mathrm{~h}$ and then treated with reagent medium or appropriate control medium for $48 \mathrm{~h}$. Cells were then incubated with tetrazolium reagent and absorbance was measured. Apoptotic activity was assayed in parallel cultures using the ApoONE Homogeneous Caspase 3/7 Apoptosis Assay (Promega, Madison, WI, USA) according to the manufacturer's instructions. ${ }^{38,39}$

\section{Hh-Responsive Luciferase Reporter Assay}

As primary HSC are not very amenable to detailed molecular manipulations, including transfection, ${ }^{23,32}$ the Hh-responsive luciferase reporter assay was performed on cultures of HSC line $8 \mathrm{~B}$ and a positive control cell line, C3H10T1/2, in replicates $(n=6-8)$ as described previously. ${ }^{40}$ Briefly, both lines were transfected with $9 \times$ Gli-binding siteluciferase plasmid and pRL-TK (Promega) along with vector or constitutively active, wild-type Smo using Lipofectamine 2000 (Invitrogen, Carlsbad, CA, USA) according to the manufacturer's recommendations. After a $3.5 \mathrm{~h}$ transfection, cells were washed twice with DMEM:Ham's F-12 (1:1) medium and then cultured overnight in DMEM:Ham's F-12 (1:1) medium. Cells were harvested $16 \mathrm{~h}$ after transfection and lysed in reporter lysis buffer (Promega). Reporter activity was determined by using the Dual-Luciferase Reporter Assay System (Promega). Activity of the Firefly luciferase reporter was normalized to the activity of a Renilla luciferase internal control for transfection efficiency.

\section{Two-Step Real-Time Reverse Transcription-Polymerase Chain Reaction (RT-PCR)}

Total RNA was extracted from whole liver, primary cells, and HSC lines with the RNeasy kit, followed by RNase-free DNase I treatment (Qiagen, Valencia, CA, USA). The primers were designed using Genbank sequences or as described previously (Table 1). ${ }^{11,34,41-43}$ For all primer pairs, specificity was confirmed by sequencing of PCR products. Amplicon products were separated by electrophoresis on a $2.0 \%$ agarose gel buffered with $0.5 \times$ TBE. Optimal annealing temperatures with appropriate melt curves were determined and PCR efficiency was confirmed above $90 \%$ for all primer sets. For each experiment, total RNA was reverse transcribed to cDNA templates and amplified using Ready-ToGo You-Prime First-Strand Beads (Amersham) with pd(N)6 first-strand cDNA primers (Amersham). For quantitative RT-PCR, $1.5 \%$ of the first-strand reaction was amplified using iQ-SYBR Green Supermix (Bio-Rad), an iCycler iQ Real-Time Detection System (Bio-Rad), and specific oligonucleotide primers for 
Table 1 RT-PCR primers for analysis

\begin{tabular}{|c|c|c|c|c|}
\hline Gene & Genbank accession number & Direction & Sequence & Amplicon size (bp) \\
\hline \multirow[t]{2}{*}{ Shh } & \multirow[t]{2}{*}{ NM_009170 } & Forward & CTGGCCAGATGTTTTCTGGT & \multirow[t]{2}{*}{117} \\
\hline & & Reverse & TAAAGGGGTCAGCTTTTTGG & \\
\hline \multirow[t]{2}{*}{ Ihh } & \multirow[t]{2}{*}{ NM_010544 } & Forward & CCGAACCTTCATCTTGGTG & \multirow[t]{2}{*}{124} \\
\hline & & Reverse & ACAGATGGAATGCGTGTGAA & \\
\hline \multirow[t]{2}{*}{ Ptc } & \multirow[t]{2}{*}{ NM_008957 } & Forward & ATGCTCCTTTCCTCCTGAAACC & \multirow[t]{2}{*}{168} \\
\hline & & Reverse & TGAACTGGGCAGCTATGAAGTC & \\
\hline \multirow{2}{*}{ Smo } & \multirow{2}{*}{ NM_176996 } & Forward & GCCTGGTGCTTATTGTGG & \multirow[t]{2}{*}{75} \\
\hline & & Reverse & GGTGGTTGCTCTTGATGG & \\
\hline \multirow[t]{2}{*}{ Gli1 } & \multirow[t]{2}{*}{ NM_010296 } & Forward & AACTCCACAGGCACACAGG & \multirow[t]{2}{*}{79} \\
\hline & & Reverse & GCTCAGGCTTCTCCTCTCTC & \\
\hline \multirow[t]{2}{*}{ Gli2 } & \multirow[t]{2}{*}{ XM_136212 } & Forward & CCATTCATAAGCGGAGCAAG & \multirow[t]{2}{*}{105} \\
\hline & & Reverse & CCAGGTCTTCCTTGAGATCG & \\
\hline \multirow{2}{*}{ Gli3 } & \multirow[t]{2}{*}{ NM_008130 } & Forward & GCTCTTCAGCAAGTGGTTCC & \multirow{2}{*}{122} \\
\hline & & Reverse & CTGTCGGCTTAGGATCTGTTG & \\
\hline \multirow{2}{*}{ Gus } & \multirow[t]{2}{*}{ NM_010368 } & Forward & GCAGTTGTGTGGGTGAATGG & 142 \\
\hline & & Reverse & GGGTCAGTGTGTTGTTGATGG & \\
\hline$\alpha-s m a$ & X06801 & Forward & TGTGTGAAGAGGAAGACAGCAC & 462 \\
\hline & & Reverse & GCACAATACCAGTTGTACGTCC & \\
\hline$M m p-2^{41}$ & NM_031054 & Forward & TGCAACCACAACCAACTACG & 705 \\
\hline & & Reverse & TCTGCGATGAGCTTAGGGAA & \\
\hline Gfap & NM_017009 & Forward & CTGGAGGTGGAGAGGGACAAT & 486 \\
\hline & & Reverse & GGACTCAAGGTCGCAGGT & \\
\hline Nestin & NM_016701 & Forward & CTCTGCTGGAGGCTGAGAAC & 120 \\
\hline & & Reverse & GGTGCTGGTCCTCTGGTATC & \\
\hline Shh & NM_000193 & Forward & GCTCGGTGAAAGCAGAGAAC & 109 \\
\hline & & Reverse & CTCAGGTCCTTCACCAGCTT & \\
\hline Ihh & NM_050846 & Forward & TCCGTCAAGTCCGAGCAC & 109 \\
\hline & & Reverse & GCCTCACGGCTGACAAGG & \\
\hline$P t c^{34}$ & NM_000264 & Forward & CCACCAGACGCTGTTTAGTCA & 72 \\
\hline & & Reverse & CGATGGAGTCCTTGCCTACAA & \\
\hline $\mathrm{Smo}^{42}$ & NM_005631 & Forward & CAGTTCCAAACATGGCAAACAG & 200 \\
\hline & & Reverse & TGCTATGTCAGGCCAATGTGA & \\
\hline Gli1 $^{42}$ & NM_005269 & Forward & TGCAGTAAAGCCTTCAGCAATG & 132 \\
\hline & & Reverse & TTTTCGCAGCGAGCTAGGAT & \\
\hline Gli2 $^{42}$ & AB007295 & Forward & CGAGAAACCCTACATCTGCAAGA & 88 \\
\hline & & Reverse & GTGGACCGTTTTCACATGCTT & \\
\hline Gli3 $^{42}$ & NM_000168 & Forward & AAACCCCAATCATGGACTCAAC & 98 \\
\hline & & Reverse & TACGTGCTCCATCCATTTGGT & \\
\hline Gus $^{43}$ & NM_000181 & Forward & CTCATTTGGAATTTTGCCGATT & 81 \\
\hline & & Reverse & CCGAGTGAAGATCCCСTTTTT & \\
\hline Gfap & NM_010277 & Forward & TCCTGGAACAGCAAAACAAG & 224 \\
\hline & & Reverse & CAGCCTCAGGTTGGTTTCAT & \\
\hline$\alpha-s m a$ & NM_007392 & Forward & GGCTCTGGGCTCTGTAAGG & 149 \\
\hline & & Reverse & CTCTTGCTCTGGGCTTCATC & \\
\hline $\operatorname{Col} 1 \alpha 2^{11}$ & BC007158 & Forward & GAACGGTCCACGATTGCATG & 167 \\
\hline & & Reverse & GGCATGTTGCTAGGCACGAAG & \\
\hline
\end{tabular}

target sequences, as well as the $\beta$-glucuronidase (Gus) housekeeping gene. The PCR parameters were as follows: denaturing at $95^{\circ} \mathrm{C}$ for $3 \mathrm{~min}$ followed by 40 cycles of denaturing at $95^{\circ} \mathrm{C}$ for $15 \mathrm{~s}$ and annealing-extension at the optimal primer temperatures for 45-60 s. Threshold cycles (Ct) were automatically calculated by the iCycler iQ Real-Time Detection System. Target gene levels in the treated cells or tissues are presented as a ratio to levels detected in the corresponding control cells or tissues, respectively, according to the $\Delta \Delta \mathrm{Ct}$ method as reported previously. ${ }^{44}$ These fold changes were determined using point and interval estimates.

\section{In Vivo Cyc Treatment}

In all, 25 mice were injected intraperitoneally with either Cyc $(15-30 \mathrm{mg} / \mathrm{kg}, \quad n=13$, Toronto
Research Chemicals, Canada) or corn oil vehicle $(n=12$, Sigma-Aldrich). The Cyc was prepared as $10 \mathrm{mg} / \mathrm{ml}$ in corn oil vehicle. No mice died following treatment. All mice were killed after $24 \mathrm{~h}$. Following euthanasia, livers were snap frozen in liquid nitrogen for subsequent RNA analysis or were fixed in formalin, paraffin-embedded, stained with hematoxylin and eosin, and histology examined by light microscopy. Given that the doses we administered were much lower (40 and 70\% less) than the previously reported standard treatment $^{34}$ and we found no overt adverse effects, we restricted our immunofluorescent staining and real-time RT-PCR analyses to mice $(n=5)$ that received the higher, $30 \mathrm{mg} / \mathrm{kg}$, dose. Hepatic RNA was extracted from two of these livers and the remaining three livers were used for immunofluorescent staining. 


\section{Statistical Analysis}

Comparisons between groups were made using Intercooled Stata 8.0 (Stata Corporation, College Station, TX, USA). Results are reported as mean \pm standard deviation (s.d.). Comparisons between groups were performed using the Student's $t$-test. Significance was accepted at the 5\% level.

\section{Results}

\section{Adult Mouse Liver Cells Possess Active Hh Signaling}

The Hh pathway is activated when Hh ligands bind to their cell surface receptor, Ptc. The ensuing intracellular cascade modulates the expression of target genes, including Ptc and Gli1. ${ }^{16}$ Based upon this cascade, Ptc-lacZ mice that carry a transgene encoding $\beta$-galactosidase driven by Hh-responsive elements in the Ptc promoter have been used to localize cellular $\mathrm{Hh}$ pathway activity. ${ }^{21,45}$ Earlier studies of these mice clearly demonstrated that mature hepatocytes and cholangiocytes in healthy adult livers lack Hh activity. ${ }^{34}$ To determine if $\mathrm{Hh}$ signaling existed in other liver cell populations, we perfused the livers of two healthy adult Ptc-lacZ mice and isolated the NPC and HSC populations by standard density gradient centrifugation. Protein extracted from the crude NPC fraction and the highly enriched HSC fraction, but not the hepatocyte fraction, exhibited $\beta$-galactosidase activity (Figure 1a), demonstrating that Hh signaling was active in cells residing within the first two liver cell fractions.

To confirm our findings by an alternative technique, we purified the HSC and NPC fractions from the livers of six healthy adult mice. We then compared the mRNA expression of Hh pathway components within the HSC fraction and the primary NPC fraction from these livers. Interestingly, the HSC fraction had a 313-fold higher expression of Shh and a seven-fold higher expression of Indian hedgehog (Ihh) than the NPC fraction, despite relatively little difference in the levels of PtC expression (Figure 1b). Thus, the freshly isolated HSC fraction produced significantly more $\mathrm{Hh}$ ligands than the NPC fraction. Further RT-PCR analysis of the HSC fraction confirmed that these cells expressed mRNA for other components of the Hh pathway, including Smo and members of the Gli family of transcription factors (Figure 1c).

\section{Activated HSC Express Hh Pathway Components}

Compared to the crude NPC fraction, the HSC fraction has been purified to eliminate most but not all blood cells, committed hepatic progenitors (ie, oval cells), macrophages, endothelial cells, and biliary epithelial cells. Conversely, the crude NPC fraction always includes some HSC. Although our
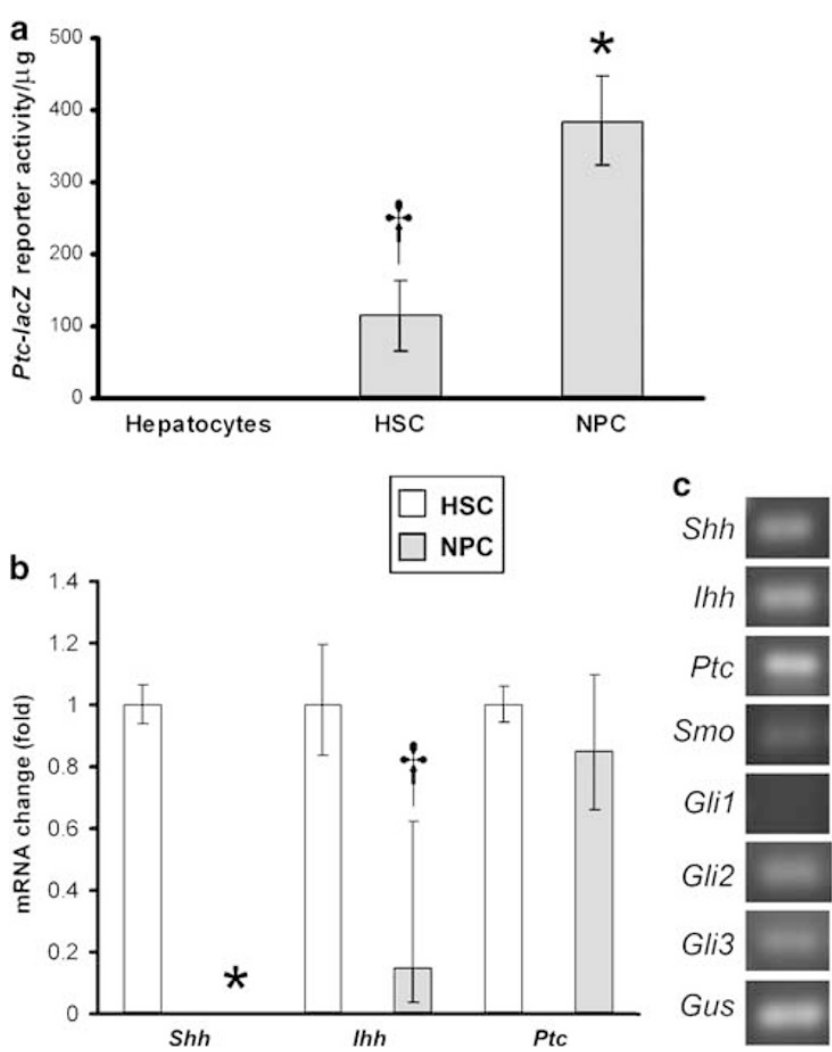

Figure $1 \mathrm{Hh}$ pathway activity in adult mouse liver. (a) Liver perfusion and density gradient centrifugation were used to purify cells from the livers of two Ptc-lacZ mice in which $\beta$-galactosidase reports Hh signaling. Proteins were extracted from the hepatocyte, hepatic stellate cell (HSC), and residual NPC fractions. Results are presented as the mean \pm s.d. of three experiments $\left({ }^{\dagger} P<0.05\right.$, ${ }^{\star} P<0.001$ ). (b) In separate experiments, liver cells were similarly isolated from six healthy adult wild-type mice. Quantitative realtime RT-PCR analysis of mRNA was performed to compare expression of Hh ligands (Shh and $I h h$ ) and receptor (PtC) in the HSC and NPC fractions $\left({ }^{\dagger} P<0.05,{ }^{*} P<0.001\right)$. (c) Agarose gel electrophoresis of the HSC RT-PCR amplicons demonstrated the Hh ligands, receptor, and signaling components (Smo, Gli2, and Gli3), as well as the Gus housekeeping gene.

studies of adult Ptc-lacZ mice and freshly isolated primary HSC from wild-type mice provided evidence that Hh-responsive cells resided in this population, it was difficult to determine whether or not Hh-responsive cells were predominately HSC. Therefore, we used three other approaches to exclude the possibility that contaminating blood cells within the HSC fractions might have biased those results.

First, we evaluated expression of Hh pathway components in primary rat HSC after the cells were culture-activated and passaged twice. This protocol unequivocally eliminates other types of cells, such as macrophages, endothelial cells, and vascular smooth muscle cells, that may have contaminated the initial primary HSC isolates. ${ }^{23,46}$ As predicted by the work of others, ${ }^{6,47}$ our primary rat HSC exhibited typical markers of myofibroblastic cells, such as $\alpha$-sma, matrix metalloproteinase-2 (Mmp-2), and 
Nestin mRNA, but lacked Gfap expression (Figure 2a). Similar to freshly isolated mouse HSC, our culture-activated rat HSC also expressed multiple components of the Hh pathway, including Shh, Ihh, Ptc, Smo, Gli1, Gli2, and Gli3 (Figure 2b).

As a second approach, we evaluated a new human HSC line, LX-2, that was shown to be virtually identical to activated human HSC by microarray analyses. ${ }^{23}$ Like culture-activated, primary rat HSC, LX-2 had low but detectable $S h$ and Ihh expression. As in rat HSC, mRNA levels of other Hh pathway components (Ptc, Smo, Gli1, Gli2, and Gli3) in the human HSC line also approximated those of Gus, a highly expressed housekeeping gene (Figure 2c). Robust Ptc expression in the LX-2 line suggested that the human cells had high endogenous $\mathrm{Hh}$ pathway activity.

Finally, we compared mRNA levels of Hh pathway components in culture-activated rat HSC and LX-2 human HSC to primary mouse HSC that were culture-activated for 7 days. We found similar patterns of Hh component expression in HSC from all three species (Figure 2c).

\section{Inhibition of Hh Signaling Abrogates Primary Hepatic Stellate Cell Activation}

Next, the influence of Hh signaling on HSC activation was assessed by comparing the effects of treatment with Cyc (a pharmacologic inhibitor of Hh signaling) with that of tomatidine (an inactive Cyc analog) ${ }^{34}$ on the spontaneous differentiation of quiescent primary murine HSC to activated myofibroblasts in vitro. Standard markers of quiescence (eg, Gfap) and activation (eg, $\alpha$-sma, and type I collagen $\alpha 2($ Col1 $\alpha 2)$ ) were evaluated by real-time RT-PCR. In control (tomatidine-treated) cultures, Gfap expression decreased (by more than 24-fold), while $\alpha$-sma and Col1 $\alpha 2$ expression increased dramatically (Figure 3a) after 7 days, and the cells enlarged and became polygonally shaped with large nuclei (Figure 3b). In contrast, typical morphologic features of activation ${ }^{30,48}$ were less frequent after 7 days of $\mathrm{Hh}$ inhibition, and most of the cells remained compact with small nuclei (Figure 3c). Immunofluorescent staining revealed markedly reduced $\alpha$-SMA in Hh-inhibited HSC (Figure 3e) compared to control HSC (Figure 3d). Systematic quantification of DAPI-stained nuclei demonstrated fewer HSC (8.1 \pm 4.7 HSC/field) in Cyc-treated cultures than in control cultures $(10.5 \pm 4.2 \mathrm{HSC} /$ field). This modest (22\%) decrease in HSC number was consistent, albeit not statistically significant $(P<0.10)$, based upon counts in 22 random $40 \times$ fields per plate. Real-time RT-PCR analysis showed that Cyc decreased $\alpha$-sma expression by $22 \%$ (data not shown) and Col1 $\alpha 2$ expression by nearly $50 \%$ (Figure $3 f$ ). Together, these findings indicated that inhibition of Hh signaling with Cyc appreciably reduced spontaneous activation/differentiation of HSC in vitro.


Figure 2 Activated rat, human, and mouse HSC from adult livers express Hh signaling components. (a) Primary HSC were isolated from the livers of healthy rats using in situ liver perfusion and density gradient centrifugation. Cells were placed in culture, passaged twice, and then harvested for RNA isolation. RT-PCR analysis was carried out to evaluate markers of stellate cell activation ( $\alpha$-sma, Mmp-2, and Nestin) and quiescence (Gfap). (b) RT-PCR analysis of these same samples also demonstrated expression of the Hh ligands (Shh and $I h h$ ), the Hh receptor (PtC), and downstream signaling components of $\mathrm{Hh}$ pathway (Smo, Gli1, Gli2, and Gli3), as well as the Gus housekeeping gene. (c) RNA was obtained from cultured primary rat HSC, the LX-2 human HSC line, and culture-activated, primary mouse HSC, and analyzed for Hh pathway expression by realtime RT-PCR. For each sample, expression of the Hh pathway components was normalized to expression of the housekeeping gene, Gus. Results in HSC from the three different species are displayed.

\section{Hh Activity Maintains Clonal Rat Hepatic Stellate Cell Lines}

Next, we evaluated two clonal rat HSC lines that were derived from the liver of a single rat that was treated with carbon tetrachloride. ${ }^{24,49-51}$ HSC line $5 \mathrm{H}$ is known to exhibit the features of a classic myofibroblast, including copious production of matrix proteins, such as type I collagen. HSC line $8 \mathrm{~B}$ is considered to be more 'stellate cell-like', 
upregulating collagen expression when exposed to transforming growth factor beta (TGF- $\beta$ ), expressing platelet-derived growth factor- $\beta$ (PDGF- $\beta$ ) receptors, and proliferating in response to PDGF. Our RT-PCR analysis demonstrated that both cell lines retained the typical features of culture-activated primary
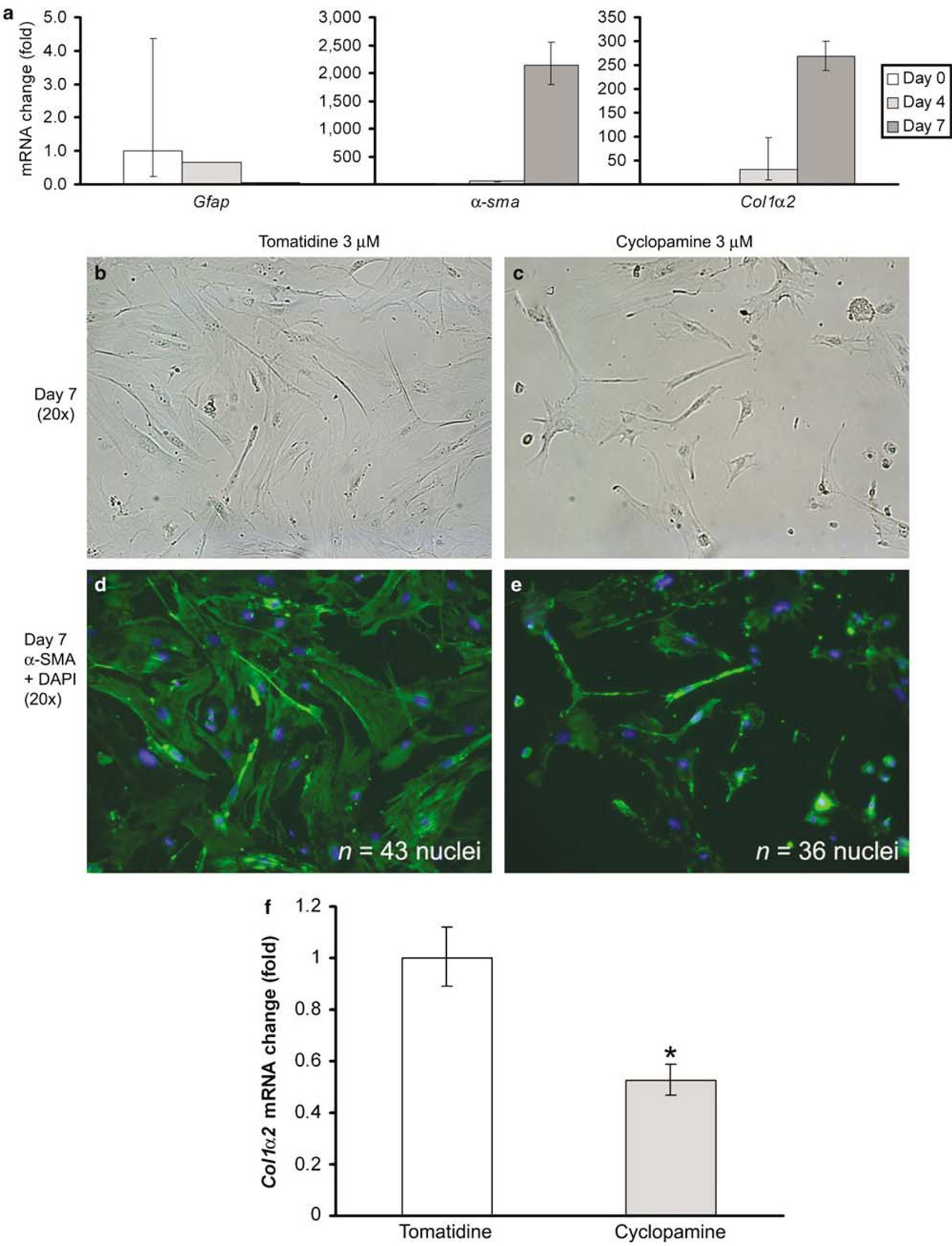
HSC. ${ }^{52}$ They expressed neural markers, including Nestin and Gfap, as well as produced mesenchymal factors, including $M m p-2$ and $\alpha$-sma (data not shown).

We evaluated these HSC clones for expression of Shh, Ihh, Ptc, Smo, and the Gli family of transcription factors. Both rat HSC lines expressed cellular components of the Hh pathway (Figure 4a). Interestingly, unlike our primary HSC and LX-2 cells which expressed both Shh and Ihh, the rat HSC lines had distinct patterns of ligand expression: one of them $(5 \mathrm{H})$ expressed Shh, while the other (8B) expressed Ihh. This finding was consistent with abundant evidence that the HSC population in adult livers is heterogeneous. ${ }^{1,4}$

To determine if expression of $\mathrm{Hh}$ pathway components was accompanied by functional pathway activity, we transfected cultures of HSC line 8B with an Hh-inducible Gli-luciferase reporter. ${ }^{40}$ Basal Gli-reporter activity in HSC line 8B was equivalent to that of a positive control cell line (C3H10T1/2) that was cotransfected with constitutively active Smo (Figure 4b). Introduction of Smo upregulated Gli-luciferase activity in the HSC 8B cells by $220 \%$ $(P<0.01)$. These findings demonstrated that our clonal HSC line not only expressed Hh signaling components but also exhibited constitutive and inducible Hh pathway activity.

As mentioned earlier, Hh signaling is known to regulate the viability of neural and myofibroblastic cells in the brain and gut. We found that $\mathrm{Hh}$ pathway inhibition slightly decreased primary HSC numbers in vitro. Therefore, we cultured the lines with recombinant Shh-N ligand or neutralizing antibody to Shh (5E1) to examine the influence of Hh pathway activity on HSC viability. The $5 \mathrm{H}$ clone that constitutively expressed $S h h$ was particularly sensitive to depletion of Shh, demonstrating striking dose-related increases in apoptotic activity (Figure 4c) and decreases in viability (Figure 4d) when cultured in the presence of Shh-neutralizing antibody (5E1). Conversely, Shh-N ligand decreased apoptotic activity (Figure 4c) and increased the viability (Figure $4 \mathrm{~d}$ ) of this line in a dose-dependent fashion. To corroborate these findings, we treated the LX-2 human HSC line with modulators of Hh signaling. Shh neutralization with 5E1 antibody increased caspase $3 / 7$ activity in LX-2 cells by up to $48 \%$ (data not shown). Therefore, activated HSC relied upon Hh ligands for optimal viability.

\section{Inhibition of Hh Signaling Maintains Quiescent HSC In Vivo}

We next investigated the effect of inhibiting $\mathrm{Hh}$ pathway activity in healthy adult mice. Mice were treated with either corn oil vehicle or a single intraperitoneal injection of Cyc 1 day before sacrifice, ${ }^{53}$ and we performed immunofluorescent staining for GFAP (Figure 5a) and $\alpha$-SMA (Figure 5b) in liver sections from representative mice. GFAPpositive and $\alpha$-SMA-positive HSC were generally quite rare (range: $0-3$ per $40 \times$ field). However, comparison of the average number of cells in 50-75 fields revealed a two-fold increase in GFAP-positive cells in the Cyc group $(P<0.05)$, with an equal and opposite (ie, $50 \%$ ) reduction in $\alpha$-SMA-positive cells $(P<0.01)$ in the same livers (Figure 5c). Real-time RT-PCR analysis showed that Cyc significantly inhibited hepatic expression of two markers of activated HSC, reducing mRNA of Nestin by $63 \%$ and $\alpha$-sma by $74 \%$, while increasing a marker of quiescent HSC, Gfap, by $78 \%$ (Figure $5 \mathrm{~d}$ ). We did not see an effect on Col1 $\alpha 2$ expression (data not shown) during this brief treatment period, and the expense of Cyc prohibited us from evaluating the effects of repeated Cyc injections on the evolution of cirrhosis. Liver weights and liver/body weight ratios were similar in the Cyc-treated group and controls, and none of the Cyc-treated mice exhibited liver injury on H\&E-stained liver sections (data not shown). Thus, a single treatment with a highly specific inhibitor of $\mathrm{Hh}$ activity rapidly upregulated a quiescent HSC marker and downregulated markers of activated HSC without adversely influencing the health or liver histology of adult mice.

\section{Discussion}

These results identify a novel mechanism, namely Hh signaling, which regulates HSC viability and activation. Using several complementary experimental approaches, we demonstrated that HSC in adult livers express Hh ligands, Ptc receptor, and several downstream components of the Hh pathway, have endogenous pathway activity, and rely upon Hh signals for activation and optimal viability.

Although Hh has not been previously considered as a potential differentiation or viability factor for HSC, our findings are consistent with abundant

Figure 3 Primary HSC activation is regulated by Hh signaling. (a) HSC were isolated and pooled from the livers of 12 healthy adult mice. The cells were cultured on plastic in serum-containing medium for up to 7 days. Cultures were harvested at different time points (days 0 , 4 , or 7) to obtain RNA for quantitative RT-PCR analysis of markers for quiescence (Gfap) and activation ( $\alpha$-sma), as well as Col1 $\alpha 2$ expression. Parallel cultures were treated with the Hh pathway inhibitor, Cyc $(3 \mu \mathrm{M})$, or an equivalent concentration of the biologically inactive analog, tomatidine, for 7 days. At the end of the treatment period, cultures were compared for cell morphology $(\times 20$ magnification) (b, c) and expression of the HSC activation marker, $\alpha$-SMA (d, e). Costaining with DAPI was carried out to demonstrate cell nuclei. (f) RNA was isolated from the day 7 cultures and real-time RT-PCR analysis was performed to evaluate Col1 22 expression $\left({ }^{*} P<0.001\right)$. 
a

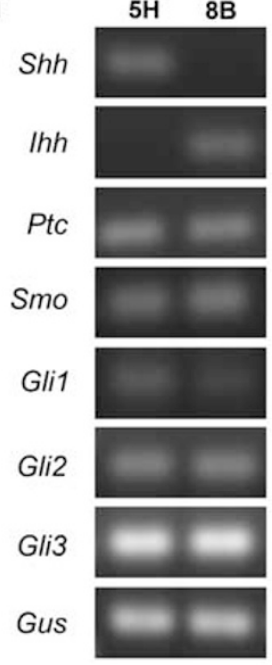

b

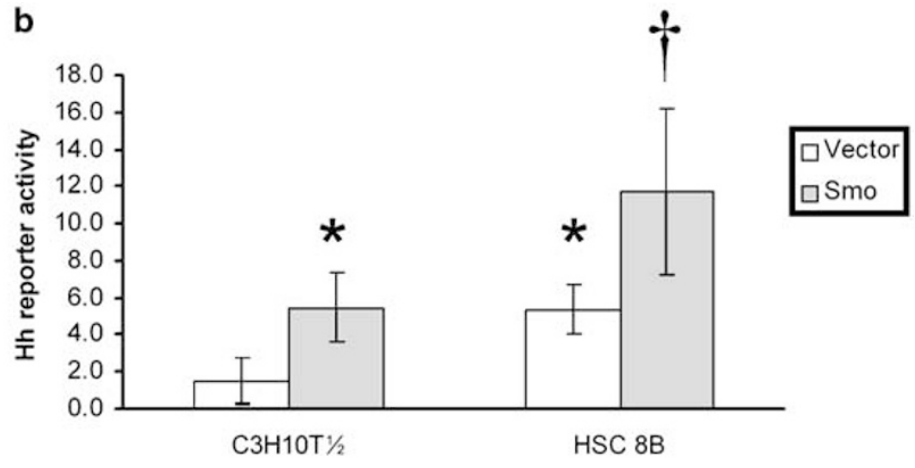

C

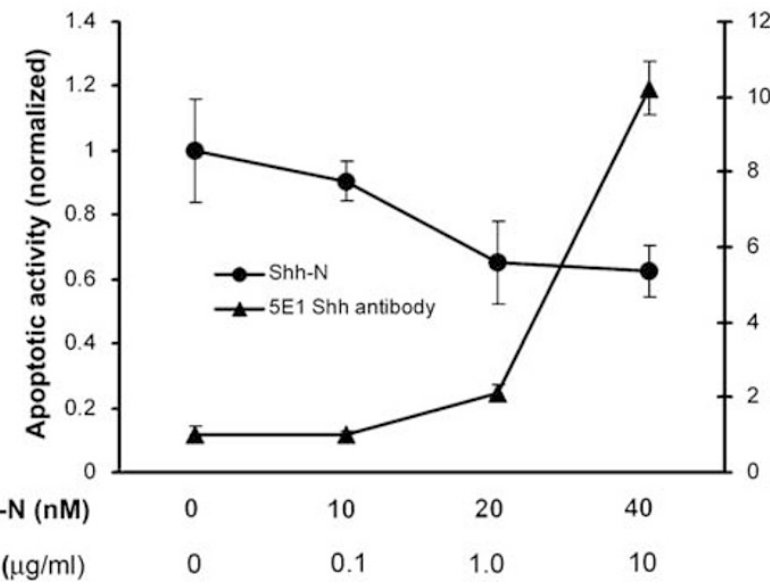

d

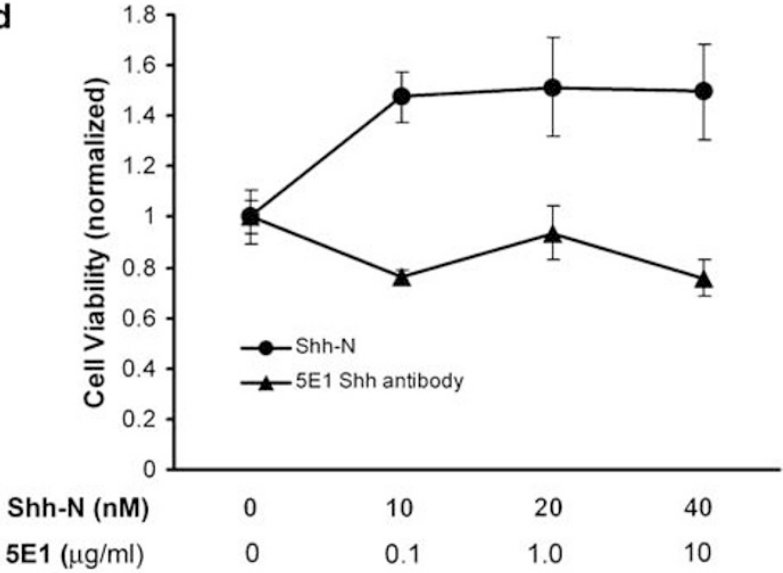

Figure 4 Clonally derived HSC lines from adult rat livers express Hh signaling components. (a) RNA was isolated from two different, clonally derived rat HSC lines that were generated from primary HSC obtained from a rat with carbon tetrachloride-induced cirrhosis. Expression of Hh pathway components was evaluated by RT-PCR. Representative agarose gel electrophoresis of RT-PCR products from HSC lines 5H and 8B demonstrated expression of Hh ligands (Shh and Ihh), receptor (PtC), and downstream signaling components (Smo, Gli1, Gli2, and Gli3), as well as the Gus housekeeping gene. (b) HSC line 8B was transiently transfected with an Hh-inducible Gliluciferase reporter alone (Vector) or with a plasmid for constitutively active Smo, an activator of Hh signaling (Smo). Results were compared to a similarly transfected positive control cell line, C3H10T1/2 ( ${ }^{*} P<0.01$ vs C3H10T1/2 + Vector, ${ }^{*} P<0.01$ vs HSC 8B + Vector). HSC line $5 \mathrm{H}$ was cultured in the presence or absence of various concentrations of an Hh pathway activator (Shh-N) or an Hh pathway inhibitor (5E1 Shh antibody) for $48 \mathrm{~h}$. Apoptotic activity was evaluated by assessing caspase $3 / 7$ activity (c). In parallel cultures, cell viability was assessed by tetrazolium salt metabolism (d).

evidence that $\mathrm{Hh}$ regulates the viability and differentiation of other cells with neural and smooth muscle-like phenotypes. Hh activity is required for the development of the enteric nervous system, ${ }^{15}$ and to establish crypt-villus patterning in developing intestinal mucosa. ${ }^{19}$ The latter process involves interaction between Hh ligand and Ptc-expressing subepithelial myofibroblasts and smooth muscle cells. In embryos, the Hh pathway plays a fairly generalized role in directing mesenchymal maturation. Shh upregulates smooth muscle actin in the mesenchyme of embryonic lung explants. ${ }^{54}$ In developing limb cartilage, Ihh induces expression of Ptc in perichondrial cells. ${ }^{55,56}$ Our current studies add to this body of work by demonstrating that quiescent and activated HSC express Hh pathway components and require pathway activity for differ- entiation to activated myofibroblasts in vitro and in vivo.

Although the Hh pathway has been most extensively studied in developing embryos, ${ }^{57}$ emerging evidence indicates that it also modulates the homeostasis of several adult tissues. This has been most clearly demonstrated in skin, where direct pharmacologic manipulation of Hh signaling has been used to improve psoriasis in adult patients. ${ }^{58}$ Mucosal expression of Hh ligand and its receptor, Ptc, has also been documented in healthy adult intestine, and pathway activity increases in an array of inflammatory gut disorders. ${ }^{20}$ Finally, excessive activation of the Hh pathway is involved in the formation of a variety of tumors in adults, ${ }^{16}$ including basal cell carcinoma, ${ }^{59}$ cholangiocarcinoma, ${ }^{34}$ malignancies of the proximal gastrointestinal tract, ${ }^{34}$ 

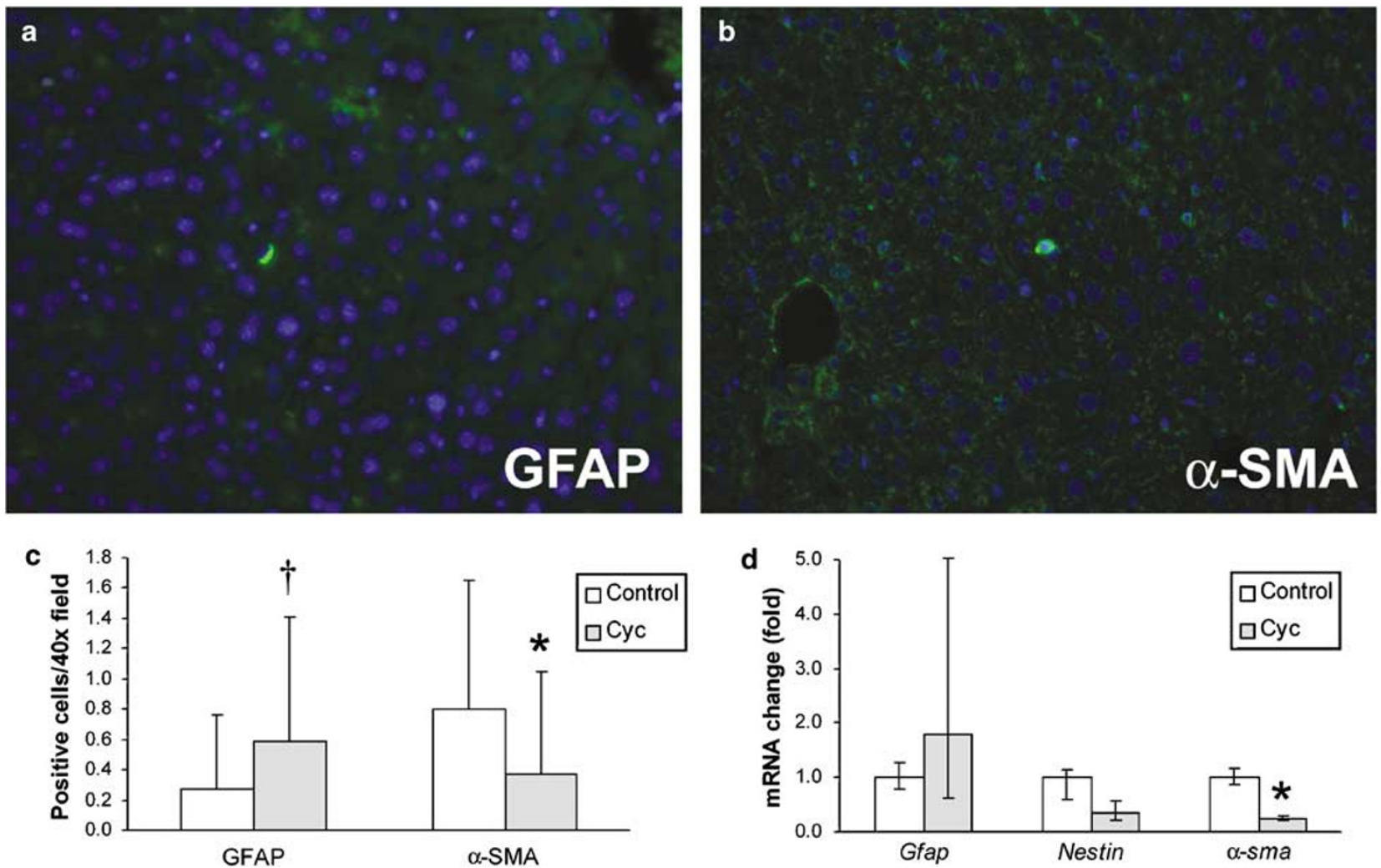

Figure $5 \mathrm{Hh}$ pathway inhibition reduces HSC differentiation in vivo without causing liver damage. Healthy adult mice were treated with a single intraperitoneal injection of Cyc or corn oil vehicle and killed $24 \mathrm{~h}$ later to obtain liver tissue. Immunofluorescence microscopy was used to quantify GFAP-positive (a) and $\alpha$-SMA-positive (b) HSC in controls and $30 \mathrm{mg} / \mathrm{kg}$ Cyc-treated group. Representative photomicrographs are shown at $\times 40$ magnification. (c) Numbers of GFAP- or $\alpha$-SMA-positive cells were counted in 50-75 fields $(\times 40$ magnification) from mice in each treatment group. Results are shown as the mean \pm s.d. $\left({ }^{\dagger} P<0.05,{ }^{*} P<0.01\right)$. (d) RNA was isolated from the remaining liver samples and analyzed by quantitative RT-PCR for markers of stellate cell quiescence (Gfap) and activation (Nestin and $\alpha$-sma) $\left({ }^{*} P<0.01\right)$.

and prostate cancer. ${ }^{60}$ Thus, there is no doubt that cells possessing $\mathrm{Hh}$ signaling persist and thrive in several adult tissues. ${ }^{57}$ Our work adds liver to the list of adult organs that harbor Hh-responsive cells, extending earlier reports that demonstrated induction of mRNA for Hh-inhibitory protein, Ptc, and Smo in whole liver extracts from cirrhotic patients. ${ }^{61-63}$ Interestingly, a large hepatic mesenchymal tumor has been reported recently in a patient with Gorlin's syndrome, which is caused by germline mutations in Ptc that result in Hh pathway activation. ${ }^{64}$ Our work suggests that resident HSC may be a source of these Hh ligands. Given that HSC accumulate in cirrhosis, which is a major risk factor for hepatocellular carcinoma, these findings also complement other work from our group which demonstrates Hh pathway activation in human hepatocarcinogenesis. ${ }^{65}$

At present, published evidence linking $\mathrm{Hh}$ activity to fibrosis is relatively indirect. However, one possible mechanism might involve the mammalian target of rapamycin (mTOR) pathway. mTOR modulates hepatic fibrosis, as demonstrated by evidence that rapamycin inhibits HSC proliferation and fibrinogenesis. ${ }^{66}$ In this regard, HSC resemble dermal fibroblasts in which transfection with small interference RNA targeted at mTOR significantly decreases collagen gene expression. ${ }^{67}$ These findings are pertinent to our work because the mTOR pathway is sensitized by Gli1, ${ }^{68}$ a known signaling component and transcriptional target of Hh signals. The possibility that Hh promotes fibrosis by activating mTOR is further supported by observations that keloid scars overexpress Gli1 and are improved by treatment with mTOR inhibitors. ${ }^{69}$ We show that, like mTOR antagonists, Cyc, an inhibitor of $\mathrm{Hh}$ signaling, decreases HSC activation and collagen gene expression. A link between $\mathrm{Hh}$ and liver fibrosis is also supported by evidence that cholesterol-lowering drugs block HSC proliferation and reduce collagen gene expression. ${ }^{70}$ This may involve inhibition of Hh signaling because Shh protein requires cholesterol modification for functional activity. ${ }^{71}$ Additional investigation of Hh-mTORcholesterol interactions will be required to substantiate or refute these theories. In any case, our findings provide compelling evidence for a previously unappreciated role for Hh signaling in HSC activation and liver fibrinogenesis.

Further work is necessary to delineate if and how Hh activity might regulate HSC biology during liver injury and repair. Much of the previous work on 
HSC has focused on their role in matrix formation during pathologic repair, such as cirrhosis. ${ }^{1}$ However, HSC are also thought to play a role in healthy remodeling of liver tissue, such as occurs following partial hepatectomy. ${ }^{72}$ Our findings in Cyc-treated mice are intriguing in this regard because they suggest that Hh signaling is necessary for the activation of quiescent HSC to activated myofibroblasts in uninjured livers. Another group has recently reported that $\mathrm{Hh}$ activity is necessary to expand pulmonary neuroendocrine cells. ${ }^{25}$ While the precise mechanisms by which resident neuroendocrine cells modulate tissue homeostasis remain obscure in both lung and liver, evidence that the $\mathrm{Hh}$ pathway is involved opens a new area for research. In addition, the identification of $\mathrm{Hh}$ inhibition as a means to maintain HSC quiescence is particularly exciting because it provides a new therapeutic target in cirrhosis. ${ }^{2,5}$

\section{Acknowledgements}

We thank Dr PA Beachy for the kind gift of adult Ptc-lacZ mice used in these studies, as well as criticisms; Dr SL Friedman for the kind gift of the LX-2 human hepatic stellate cell line; Drs SS Karhadkar and J Kim for technical assistance, criticisms, and discussion; and DF Sandler for assistance with manuscript preparation. This work was supported by the National Institutes of Health grants RO1 AA010154 (AMD), RO1 DK053792 (AMD), RO1 AA012059 (AMD), RO1 AA01541 (MR), and T32 DK007713 (JKS).

\section{References}

1 Bataller R, Brenner DA. Liver fibrosis. J Clin Invest 2005;115:209-218.

2 Fallowfield JA, Iredale JP. Targeted treatments for cirrhosis. Expert Opin Ther Targets 2004;8:423-435.

3 Sato M, Suzuki S, Senoo H. Hepatic stellate cells: unique characteristics in cell biology and phenotype. Cell Struct Funct 2003;28:105-112.

4 Magness ST, Bataller R, Yang L, et al. A dual reporter gene transgenic mouse demonstrates heterogeneity in hepatic fibrogenic cell populations. Hepatology 2004; 40:1151-1159.

5 Friedman SL. Stellate cells: a moving target in hepatic fibrogenesis. Hepatology 2004;40:1041-1043.

6 Niki T, Pekny M, Hellemans K, et al. Class VI intermediate filament protein nestin is induced during activation of rat hepatic stellate cells. Hepatology 1999;29:520-527.

7 Roskams T, Cassiman D, De Vos R, et al. Neuroregulation of the neuroendocrine compartment of the liver. Anat Rec A Discov Mol Cell Evol Biol 2004;280: 910-923.

8 Oben JA, Roskams T, Yang S, et al. Hepatic fibrogenesis requires sympathetic neurotransmitters. Gut 2004;53: 438-445.
9 Oben JA, Diehl AM. Sympathetic nervous system regulation of liver repair. Anat Rec A Discov Mol Cell Evol Biol 2004;280:874-883.

10 Oben JA, Yang S, Lin H, et al. Acetylcholine promotes the proliferation and collagen gene expression of myofibroblastic hepatic stellate cells. Biochem Biophys Res Commun 2003;300:172-177.

11 Oben JA, Yang S, Lin H, et al. Norepinephrine and neuropeptide $\mathrm{Y}$ promote proliferation and collagen gene expression of hepatic myofibroblastic stellate cells. Biochem Biophys Res Commun 2003;302: 685-690.

12 Bataller R, Sancho-Bru P, Gines P, et al. Activated human hepatic stellate cells express the renin-angiotensin system and synthesize angiotensin II. Gastroenterology 2003;125:117-125.

13 Reynaert H, Rombouts K, Vandermonde A, et al. Expression of somatostatin receptors in normal and cirrhotic human liver and in hepatocellular carcinoma. Gut 2004;53:1180-1189.

14 Potter JJ, Womack L, Mezey E, et al. Transdifferentiation of rat hepatic stellate cells results in leptin expression. Biochem Biophys Res Commun 1998;244: 178-182.

$15 \mathrm{Fu} \mathrm{M}$, Lui VC, Sham MH, et al. Sonic hedgehog regulates the proliferation, differentiation, and migration of enteric neural crest cells in gut. J Cell Biol 2004;166:673-684.

16 Taipale J, Beachy PA. The Hedgehog and Wnt signalling pathways in cancer. Nature 2001;411:349-354.

17 Rafuse VF, Soundararajan P, Leopold C, et al. Neuroprotective properties of cultured neural progenitor cells are associated with the production of sonic hedgehog. Neuroscience 2005;131:899-916.

18 van den Brink GR, Bleuming SA, Hardwick JC, et al. Indian Hedgehog is an antagonist of Wnt signaling in colonic epithelial cell differentiation. Nat Genet 2004; $36: 277-282$.

19 Madison BB, Braunstein K, Kuizon E, et al. Epithelial hedgehog signals pattern the intestinal crypt-villus axis. Development 2005;132:279-289.

20 Nielsen CM, Williams J, van den Brink GR, et al. Hh pathway expression in human gut tissues and in inflammatory gut diseases. Lab Invest 2004;84:1631-1642.

21 Goodrich LV, Milenkovic L, Higgins KM, et al. Altered neural cell fates and medulloblastoma in mouse patched mutants. Science 1997;277:1109-1113.

22 Chen JK, Taipale J, Cooper MK, et al. Inhibition of Hedgehog signaling by direct binding of cyclopamine to Smoothened. Genes Dev 2002;16:2743-2748.

$23 \mathrm{Xu}$ L, Hui AY, Albanis E, et al. Human hepatic stellate cell lines, LX-1 and LX-2: new tools for analysis of hepatic fibrosis. Gut 2005;54:142-151.

24 Greenwel P, Schwartz M, Rosas M, et al. Characterization of fat-storing cell lines derived from normal and CCl4-cirrhotic livers. Differences in the production of interleukin-6. Lab Invest 1991;65:644-653.

25 Watkins DN, Berman DM, Burkholder SG, et al. Hedgehog signalling within airway epithelial progenitors and in small-cell lung cancer. Nature 2003; 422:313-317.

26 Ramm GA. Isolation and culture of rat hepatic stellate cells. J Gastroenterol Hepatol 1998;13:846-851.

27 Friedman SL, Rockey DC, McGuire RF, et al. Isolated hepatic lipocytes and Kupffer cells from normal human liver: morphological and functional characteristics in primary culture. Hepatology 1992;15:234-243. 
28 Oben JA, Roskams T, Yang S, et al. Norepinephrine induces hepatic fibrogenesis in leptin deficient ob/ob mice. Biochem Biophys Res Commun 2003;308: 284-292.

29 Reeves HL, Friedman SL. Activation of hepatic stellate cells-a key issue in liver fibrosis. Front Biosci 2002;7:d808-d826.

30 She H, Xiong S, Hazra S, et al. Adipogenic transcriptional regulation of hepatic stellate cells. J Biol Chem 2005;280:4959-4967.

31 Armendariz-Borunda J, Greenwel P, Rojkind M. Kupffer cells from CC1(4)-treated rat livers induce skin fibroblast and liver fat-storing cell proliferation in culture. Matrix 1989;9:150-158.

32 Lee JS, Kang Decker N, Chatterjee S, et al. Mechanisms of nitric oxide interplay with Rho GTPase family members in modulation of actin membrane dynamics in pericytes and fibroblasts. Am J Pathol 2005;166: 1861-1870.

33 Ericson J, Morton S, Kawakami A, et al. Two critical periods of Sonic Hedgehog signaling required for the specification of motor neuron identity. Cell 1996;87: 661-673.

34 Berman DM, Karhadkar SS, Maitra A, et al. Widespread requirement for Hedgehog ligand stimulation in growth of digestive tract tumours. Nature 2003;425: 846-851.

35 Skalli O, Ropraz P, Trzeciak A, et al. A monoclonal antibody against alpha-smooth muscle actin: a new probe for smooth muscle differentiation. J Cell Biol 1986;103:2787-2796.

36 Castellano B, Gonzalez B, Jensen MB, et al. A double staining technique for simultaneous demonstration of astrocytes and microglia in brain sections and astroglial cell cultures. J Histochem Cytochem 1991;39: 561-568.

37 Itano N, Atsumi F, Sawai T, et al. Abnormal accumulation of hyaluronan matrix diminishes contact inhibition of cell growth and promotes cell migration. Proc Natl Acad Sci USA 2002;99:3609-3614.

38 Sudarshan S, Holman DH, Hyer ML, et al. In vitro efficacy of Fas ligand gene therapy for the treatment of bladder cancer. Cancer Gene Ther 2005;12:12-18.

39 Takao T, Kumagai C, Hisakawa N, et al. Effect of 17beta-estradiol on tumor necrosis factor-alpha-induced cytotoxicity in the human peripheral T lymphocytes. J Endocrinol 2005;184:191-197.

40 Merchant M, Vajdos FF, Ultsch M, et al. Suppressor of fused regulates Gli activity through a dual binding mechanism. Mol Cell Biol 2004;24:86278641.

41 Murota H, Hamasaki Y, Nakashima T, et al. Disruption of tumor necrosis factor receptor p55 impairs collagen turnover in experimentally induced sclerodermic skin fibroblasts. Arthritis Rheum 2003;48:1117-1125.

42 Katayam M, Yoshida K, Ishimori H, et al. Patched and smoothened mRNA expression in human astrocytic tumors inversely correlates with histological malignancy. J Neurooncol 2002;59:107-115.

43 Hung CJ, Ginzinger DG, Zarnegar R, et al. Expression of vascular endothelial growth factor-C in benign and malignant thyroid tumors. J Clin Endocrinol Metab 2003;88:3694-3699.

44 Livak KJ, Schmittgen TD. Analysis of relative gene expression data using real-time quantitative PCR and the 2(-Delta Delta C(T)) method. Methods 2001;25: $402-408$.
45 Pepinsky RB, Shapiro RI, Wang S, et al. Long-acting forms of Sonic hedgehog with improved pharmacokinetic and pharmacodynamic properties are efficacious in a nerve injury model. J Pharm Sci 2002;91: 371-387.

46 Geerts A, Greenwel P, Cunningham M, et al. Identification of connective tissue gene transcripts in freshly isolated parenchymal, endothelial, Kupffer and fatstoring cells by northern hybridization analysis. J Hepatol 1993;19:148-158.

47 Knittel T, Aurisch S, Neubauer K, et al. Cell-typespecific expression of neural cell adhesion molecule (N-CAM) in Ito cells of rat liver. Up-regulation during in vitro activation and in hepatic tissue repair. Am J Pathol 1996;149:449-462.

48 Friedman SL, Wei S, Blaner WS. Retinol release by activated rat hepatic lipocytes: regulation by Kupffer cell-conditioned medium and PDGF. Am J Physiol 1993;264:G947-G952.

49 Greenwel P, Rubin J, Schwartz M, et al. Liver fatstoring cell clones obtained from a CCl4-cirrhotic rat are heterogeneous with regard to proliferation, expression of extracellular matrix components, interleukin-6, and connexin 43. Lab Invest 1993;69:210-216.

50 Rojkind M, Novikoff PM, Greenwel P, et al. Characterization and functional studies on rat liver fat-storing cell line and freshly isolated hepatocyte coculture system. Am J Pathol 1995;146:1508-1520.

51 Inagaki Y, Truter S, Greenwel P, et al. Regulation of the alpha 2(I) collagen gene transcription in fat-storing cells derived from a cirrhotic liver. Hepatology 1995; 22:573-579.

52 Friedman SL. Hepatic stellate cells. Prog Liver Dis 1996;14:101-130.

53 Taipale J, Chen JK, Cooper MK, et al. Effects of oncogenic mutations in Smoothened and Patched can be reversed by cyclopamine. Nature 2000;406: 1005-1009.

54 Weaver M, Batts L, Hogan BL. Tissue interactions pattern the mesenchyme of the embryonic mouse lung. Dev Biol 2003;258:169-184.

55 Linsenmayer TF, Hendrix MJ, Little CD. Production and characterization of a monoclonal antibody to chicken type I collagen. Proc Natl Acad Sci USA 1979; 76:3703-3707.

56 Koyama E, Leatherman JL, Noji S, et al. Early chick limb cartilaginous elements possess polarizing activity and express hedgehog-related morphogenetic factors. Dev Dyn 1996;207:344-354.

57 Hooper JE, Scott MP. Communicating with hedgehogs. Nat Rev Mol Cell Biol 2005;6:306-317.

58 Tas S, Avci O. Rapid clearance of psoriatic skin lesions induced by topical cyclopamine. A preliminary proof of concept study. Dermatology 2004;209:126-131.

59 Xie J, Murone M, Luoh SM, et al. Activating Smoothened mutations in sporadic basal-cell carcinoma. Nature 1998;391:90-92.

60 Karhadkar SS, Bova GS, Abdallah N, et al. Hedgehog signalling in prostate regeneration, neoplasia and metastasis. Nature 2004;431:707-712.

61 Shackel NA, McGuinness PH, Abbott CA, et al. Identification of novel molecules and pathogenic pathways in primary biliary cirrhosis: cDNA array analysis of intrahepatic differential gene expression. Gut 2001;49:565-576.

62 Shackel NA, McGuinness PH, Abbott CA, et al. Insights into the pathobiology of hepatitis $\mathrm{C}$ virus- 
associated cirrhosis: analysis of intrahepatic differential gene expression. Am J Pathol 2002;160:641-654.

63 Shackel NA, McGuinness PH, Abbott CA, et al. Novel differential gene expression in human cirrhosis detected by suppression subtractive hybridization. Hepatology 2003;38:577-588.

64 Koch CA, Chrousos GP, Chandra R, et al. Two-hit model for tumorigenesis of nevoid basal cell carcinoma (Gorlin) syndrome-associated hepatic mesenchymal tumor. Am J Med Genet 2002;109:74-76.

65 Sicklick JK, Jayaraman A, Kannangai R, et al. Hedgehog signaling correlates with hepatocellular carcinoma progression. J Clin Oncol 2005;23:9610 (abstract 30554).

66 Zhu J, Wu J, Frizell E, et al. Rapamycin inhibits hepatic stellate cell proliferation in vitro and limits fibrogenesis in an in vivo model of liver fibrosis. Gastroenterology 1999;117:1198-1204.

67 Shegogue D, Trojanowska M. Mammalian target of rapamycin positively regulates collagen type I produc- tion via a phosphatidylinositol 3-kinase-independent pathway. J Biol Chem 2004;279:23166-23175.

68 Louro ID, McKie-Bell P, Gosnell H, et al. The zinc finger protein GLI induces cellular sensitivity to the mTOR inhibitor rapamycin. Cell Growth Differ 1999; 10:503-516.

69 Kim A, DiCarlo J, Cohen C, et al. Are keloids really 'gli-loids'?: high-level expression of gli-1 oncogene in keloids. J Am Acad Dermatol 2001;45:707-711.

70 Rombouts K, Kisanga E, Hellemans K, et al. Effect of HMG-CoA reductase inhibitors on proliferation and protein synthesis by rat hepatic stellate cells. J Hepatol 2003;38:564-572.

71 Porter JA, Young KE, Beachy PA. Cholesterol modification of hedgehog signaling proteins in animal development. Science 1996;274:255-259.

72 Balabaud C, Bioulac-Sage P, Desmouliere A. The role of hepatic stellate cells in liver regeneration. J Hepatol 2004;40:1023-1026. 